\title{
BMJ Open A study protocol for a randomised controlled trial of an interactive web- based intervention: CancerCope
}

\author{
Suzanne K Chambers, ${ }^{1,2,3,4,5}$ Lee Ritterband, ${ }^{6}$ Frances Thorndike, ${ }^{6}$ Lisa Nielsen, ${ }^{2}$ \\ Joanne F Aitken, ${ }^{1,2}$ Samantha Clutton, ${ }^{2}$ Paul Scuffham, ${ }^{7}$ Philippa Youl, ${ }^{2,8,9}$ \\ Bronwyn Morris, ${ }^{10}$ Peter Baade, ${ }^{2}$ Jeffrey Dunn ${ }^{1,2,5,11}$
}

To cite: Chambers SK, Ritterband L, Thorndike F, et al. A study protocol for a randomised controlled trial of an interactive web-based intervention: CancerCope. BMJ Open 2017;7:e017279. doi:10.1136/ bmjopen-2017-017279

- Prepublication history for this paper is available online. To view these files please visit the journal online (http://dx.doi. org/10.1136/bmjopen-2017017279)

Received 13 April 2017 Revised 21 April 2017 Accepted 26 April 2017

CrossMark

For numbered affiliations see end of article.

\section{Correspondence to}

Professor Suzanne K Chambers; suzanne.chambers@griffith. edu.au

\begin{abstract}
Introduction Approximately $35 \%$ of patients with cancer experience clinically significant distress, and unmet psychological supportive care needs are prevalent. This study describes the protocol for a randomised controlled trial (RCT) to assess the efficacy and cost-effectiveness of an internet-based psychological intervention for distressed patients with cancer.
\end{abstract}

Methods and analysis In phase I, the intervention was developed on an interactive web platform and pilot tested for acceptability using a qualitative methodology with 21 patients with cancer. Phase II is an RCT underway with patients with or at risk of elevated psychological distress comparing: (1) static patient education website with (2) individualised webdelivered cognitive behavioural intervention (CancerCope). Participants were recruited through the Queensland Cancer Registry and Cancer Council Helpline and met the following inclusion criteria: (1) recently diagnosed with cancer; (2) able to read and speak English; (3) no previous history of head injury, dementia or psychiatric illness; (4) no other concurrent cancer; (5) phone and internet access; (5) scored $\geq 4$ on the Distress Thermometer. Participants are assessed at four time points: baseline/recruitment and 2, 6 and 12 months after recruitment and intervention commencement. Of the 163 participants recruited, $50 \%$ met caseness for distress. The area of highest unmet supportive care needs were psychological followed by physical and daily living needs. Primary outcomes are psychological and cancer-specific distress and unmet psychological supportive care needs. Secondary outcomes are positive adjustment, quality of life and cost-effectiveness.

Ethics and dissemination Ethical approval was obtained from the Griffith University Human Research Ethics Committee (Approval: PSY/70/13/HREC) and the Metro South Human Research Ethics Committee (HREC/13/ QPAH/601). All participants provide informed consent prior to taking part in the study. Once completed, this study will provide recommendations about the efficacy of web-based cognitive behavioural interventions to facilitate better psychosocial adjustment for people with cancer. Trial registration number ANZCTR (ACTRN12613001026718).

\section{INTRODUCTION}

In Australia in 2014, approximately 123900 new cases of cancer were diagnosed, and in

\section{Strengths and limitations of this study}

- This study will be conducted as a randomised controlled trial testing a web-based psychological intervention to assist distressed patients with cancer.

- Patients with melanoma and colorectal cancer were specifically targeted, and there is a gap in knowledge about effective psychosocial interventions for these patients.

- The use of the web-based delivery method means that should the intervention prove effective, it can be disseminated on a population level at very low cost.

- Web-based interventions rely on the patient having ready access to the internet and sufficient web literacy to use the programme.

2009, over 370000 Australians were within 5 years of a diagnosis of cancer. ${ }^{1}$ The majority of people diagnosed with cancer adjust well over time; however, around $35 \%$ of cancer survivors experience clinically important psychological distress that may persist or even intensify over time, ${ }^{23}$ with an increased risk of suicide in the first 12 months after diagnosis ${ }^{4}$ and for people with advanced cancer. ${ }^{56}$

Psychosocial care is now well recognised as an integral part of best practice oncology care, and various groups in North America and Australia have developed clinical practice guidelines providing recommendations for such care ${ }^{7-9}$ However, despite this, the provision of evidence-based psychosocial care for patients with cancer is far from universal. ${ }^{1011}$ In Australia, unmet psychological supportive care needs in patients with cancer are highly prevalent, with up to $89 \%$ of people with cancer reporting unmet need. ${ }^{12}$ For example, in one study of Australian breast cancer survivors, approximately one-quarter of women had unmet needs for help with cancer recurrence and stress; and clinically anxious survivors reported over three times as many 
unmet needs as those who were not anxious. ${ }^{13} 14$ Psychosocial care services accessible across the illness experience are urgently needed and these will need to be community-based if they are to be accessible beyond the acute treatment setting.

\section{Community-based approaches to psychosocial intervention}

Community-based organisations are important providers of support services for patients with cancer, many of whom provide tele-based information services or helplines as a front line. ${ }^{1516}$ The Helpline service in Australia is conducted by state Cancer Councils in each jurisdiction staffed by nurses and allied health professionals who have experience and/or qualifications in oncology and additional training in psychosocial support. ${ }^{17}$ However, helplines are relatively staff/resource intensive and so have limited capacity to meet the needs of the cancer population into the future and provide brief emotional support and patient education rather than directed psychological therapies. As the cancer burden increases, unless an intervention approach is developed that can be scaled to population level, there is little hope that existing health services will meet psychosocial care needs for this target group.

A web-based approach may provide an answer. The internet overcomes many of the barriers associated with traditional face-to-face therapy, ${ }^{18}{ }^{19}$ such as the need to travel to receive therapy and the inconvenience of scheduling appointments. Internet-based interventions may also reduce the total time of treatment, as patients would not have to delay treatment while waiting for specialty clinic appointments, but could instead start and proceed through the intervention at the recommended pace. This approach also promotes self-management, whereby individuals monitor their condition and use the appropriate steps to achieve a satisfactory quality of life (QoL) ${ }^{20}$ Self-management interventions can improve mood across a range of chronic illnesses ${ }^{20}$ and for patients with cancer with subthreshold depression that have been found to lower the later incidence of major depression..$^{21}$ Therefore, interventions based on self-management have great potential as cost-effective psychological care, as well as the advantages of equity, choice and accessibility. ${ }^{22}$

Accordingly, we proposed a web-based self-help intervention to assist people experiencing distress after a cancer diagnosis. In phase I, the intervention was developed on an interactive web platform and pilot tested for acceptability. Phase II now underway is a randomised controlled trial (RCT) with patients with cancer who either have or are at risk of having elevated psychological distress and will compare: (1) a static patient education website and (2) an individualised web-delivered cognitive behavioural intervention (CancerCope). This paper reports the development of the trial protocol, study implementation and sociodemographic and clinical characteristics of participants, including psychological and cancer-specific distress, unmet supportive care needs and QoL.

\section{METHODS AND ANALYSIS Phase I}

The CancerCope programme was developed based on a five-session telephone-based cognitive behavioural therapy intervention ${ }^{23}{ }^{24}$ and modified with six cores covering: stress reduction, problem solving, cognitive challenging and improving well-being. The cores consist of educational information and expert videos from

\begin{tabular}{ll}
\hline Table 1 Overview of core components in CancerCope intervention \\
\hline Core & Objectives \\
\hline Core 1-The Cancer Journey & Recognise and understand reactions to cancer diagnosis. \\
& Learn about relaxation exercise, how to do it and how it can help to cope with stressful \\
Core 2-Understanding Stress & situations. \\
& Learn about the body's natural response to threat or change. \\
& Recognise early warning signs of stress in the body. \\
Core 3-Managing Worry & Learn to identify, and better manage, unhelpful thoughts. \\
& Learn how to open up to difficult thoughts and feelings. \\
& Understand mindfulness and how it can help to manage worry. \\
& Learn how to practice mindfulness. \\
Core 4-Tackling Problems & Learn steps for successful problem solving. \\
& Learn tips to guide making difficult decisions. \\
Core 5-Taking Care & Be reminded of the importance of looking after well-being. \\
& Learn about the potential benefits of exercise and healthy eating. \\
& Learn tips for improving sleep. \\
& Learn ways to manage fatigue. \\
& Make plans for coping with future challenges. \\
& L Identify values and goals for the future.
\end{tabular}


psychologists, as well as stories and videos about four fictional characters on their cancer journey to illustrate the different experiences of others (see table 1).

The programme has high levels of interactivity including quizzes, online diaries and games to increase user engagement, and systems to encourage use and self-management including personalised email reminders, follow-up and feedback. Cancer Council Queensland (CCQ) counsellors are alerted if a user is distressed or at high risk of distress (based on data input), triggering the need for contact. Content is tailored to the user's needs as determined by their input, including assigned behavioural homework supported by interactive components. Components that target challenges associated with cancer treatments (eg, pain, sleep disturbance, fatigue) are additionally selected if relevant. Cores are completed weekly over a 6-week period with ongoing access to the programme for 12 months.

The CancerCope programme was pilot-tested with 21 high-distress patients with cancer recruited from the CCQ Cancer Helpline, a telephone information and support service in which callers are routinely screened for distress using the Distress Thermometer (DT) ${ }^{9}$, in OctoberNovember 2014, with 2-month follow-up being completed early February 2015. Callers indicate on a scale of 0 to 10 how much distress they have been experiencing in the past week including the current day. Higher scores indicate greater distress. Inclusion criteria for phase I were that participants must: (1) be recently diagnosed with cancer; (2) be able to read and speak English; (3) have no previous history of head injury, dementia or psychiatric illness; (4) have no other concurrent cancer and (5) have phone and internet access.

Callers to the Cancer Helpline who met the selection criteria and who scored $\geq 4$ on the $\mathrm{DT}^{9}$ were offered entry into the study by the operator at the time of the call. Once verbal permission to be contacted was obtained, the operator provided the Research Team with participant contact details. Callers were then contacted by the project staff to complete recruitment and organise access to the CancerCope programme. For phase I, access to the programme was provided for 6 weeks. Baseline assessments were conducted via telephone at the time of recruitment and via online at 2 months postrecruitment. Baseline and follow-up assessments contained the same measures as in phase II (as detailed in the Measures section under Phase II). Semistructured interviews were conducted at the 2-month assessment point to improve understanding of the nature of the intervention, assess specific components/tools delivered and the mechanisms of change and provide an in-depth analysis of patients' responses to the intervention. These interviews were audiotaped, transcribed verbatim and analysed using thematic analysis.

Of the 21 participants recruited for phase I, 18 were women and 3 were men, with an average age of 56 years (range 39-73 years). The most common cancer types were breast (8), lung (3) and prostate (3), with other cancer types including endometrial, lymphoma, ovarian, rectal and renal cancer. Sixteen of the 21 participants accessed the intervention. All 21 participants completed baseline assessment, with 17 participants completing the 2-month follow-up assessment. A total of $57.6 \%$ participants met caseness on the Brief Symptom Inventory 18 (BSI 18) for clinically significant psychological distress (as indicated by a score of $\geq 57$ for either total Global Severity Index (GSI) t-score or two subscale t-scores) ${ }^{25}$ at baseline, compared with $58.8 \%$ at 2 months.

The qualitative interviews at 2 months indicated that participants found the relaxation components, personal stories and psychologist videos helpful, and the site easy to use.

"I was having, you know, anxiety and stuff and it was just, the relaxation stuff was helping me with it...just listening to other people, what they go through and just realising that you're not alone..."

"I liked watching the videos and hearing from people."

"Easy to get into and understandable."

"I thought it [CancerCope] was quite informative, it was factual, and at least I felt like it was coming from a reliable source.”

Reasons given for not using the programme included being too unwell/busy with treatment, and already had enough support.

"A lot of days I just felt sick, either sick or tired and that wasn't one of the things I thought of fitting in the day I guess.".

"I was worried about my treatment, that was the main thing on my mind... Now I'm - now I'm into my treatment I think I'm more able...it (CancerCope) has been on my mind, I must go back and do that, but yeah, I've just kept putting it on the backburner."

"I've had so much on, I've been in hospital...Yeah, look, I'm coping, I'm coping fantastic. I've got heaps of support from my church and from my friends."

Suggestions for change included less reading and making it more specific to other cancers.

"There's lots of reading to do though... and for me now...I'm just ready to move on."

"There's so many different cancers out there, maybe customise the program for the type of cancer each person has...I think people...could lose interest because... a lot of the study might not relate to you."

Based on the pilot, content in the CancerCope programme was reduced and simplified where possible, and some text converted to a video format.

\section{Phase II}

For the trial phase of the project, it is hypothesised that 2, 6 and 12 months after recruitment and commencement of the intervention: 
1. Relative to participants receiving patient education, participants receiving CancerCope will report significantly less anxiety and depression, less cancerspecific distress, lower unmet psychological supportive care needs, higher positive adjustment and improved QoL.

2. That from a health sector perspective, the incremental cost-utility ratio from CancerCope will be compared with a threshold of $\$ 50000$ per quality-adjusted lifeyear (QALY) to evaluate the effectiveness of the intervention for patients who have, or are at risk of, elevated psychological distress.

\section{Group condition}

Patient education

Access to a static website containing information that covers stress management skills, problem-solving approaches to cancer-related concerns and patient education about a healthy lifestyle to promote wellness and optimise QoL.

Individualised web-delivered cognitive behavioural intervention Structured internet delivery of an individualised cognitive behavioural intervention as described previously.

\section{Participants}

Recruitment was undertaken through: (1) the Queensland Cancer Registry (QCR), a population-based register of cancer diagnoses in Queensland, and (2) the CCQ Cancer Helpline, a telephone information and support service in which callers are routinely screened for distress.

For recruitment through the QCR, clinicians were approached for permission to contact their patients about the study. Where the doctor had given permission for contact, those patients are contacted for consent. Informed written consent was obtained before study commencement and data collection. For recruitment through the Cancer Helpline, callers who met selection criteria were offered entry into the study by the Helpline operator at the time of the call and were contacted by project staff for consent to be in the study (see figure 1).

Inclusion criteria for QCR recruitment were that participants must: (1) have been recently diagnosed with melanoma or colorectal cancer (patients will be within 6 months postdiagnosis at recruitment); (2) be able to read and speak English; (3) have no previous history of head injury, dementia or psychiatric illness; (4) have no other concurrent cancer; (5) have phone and internet access; and (6) have physician clearance to participate in the study. The diagnosing clinician assisted in determining eligibility as part of the consent process through the QCR described above. Patients were screened for distress using the $\mathrm{DT}^{9}$ during the recruitment process and those patients who scored $\geq 4$ (indicating high distress or risk of high distress) were eligible.

Eligible individuals recruited through the Cancer Helpline were all adult callers who had been diagnosed with cancer, scored $\geq 4$ on the $\mathrm{DT}^{9}$ and met inclusion criteria 2 to 5 above.

\section{Study integrity}

Ethical approval was obtained from the Griffith University Human Research Ethics Committee (Approval: PSY/70/13/HREC) and the Metro South Human Research Ethics Committee (HREC/13/QPAH/601). The study design was guided by the CONSORT statement. ${ }^{26}$ Randomisation occurred following baseline assessment. Project staff tracking assessments were blinded to condition. Randomisation occurred in blocks of 10 , with each condition randomly generated five times within each block to ensure an unpredictable allocation sequence with equal numbers of participants in each group at the completion of each block. This sequence was undertaken by the project manager and concealed from investigators. All analyses will be conducted on the basis of intention to treat. As the web-based intervention is fully standardised, there are no variations from user to user, except with regards to completion of all components which is recorded and will be included in analyses.

\section{Measures}

A series of previously validated and reliable self-report measures are administered at four time points: baseline/ recruitment and at 2, 6 and 12 months after recruitment and intervention commencement. Baseline assessments are conducted by telephone prior to randomisation, and include background variables and economic analysis information. Follow-up assessments are conducted through online questionnaires accessed through the web support programme. Data are stored securely on project databases and only accessible to research personnel trained in confidentiality and privacy procedures.

As is standard practice for eHealth interventions, participants are unable to access the CancerCope programme while their assessment is due. Participants are notified by email that their follow-up assessment is due and that, once their questionnaire is completed, they can continue on in the CancerCope programme. They are also advised of CCQ supportive care services available to them, and that they can contact the Project Manager should they need support.

If after 3 days the follow-up assessment has not been completed, an email reminder is sent to the participant. If after 7 days the follow-up assessment has not been completed, the participant receives a follow-up call reminding them to complete their online assessment. The participant regains access to the intervention immediately after this phone call regardless of assessment completion. Ten attempts are made to contact the participant, after which the assessment is marked as missed and the participant regains access to the intervention.

Primary outcomes are psychological and cancer-specific distress and unmet supportive care needs. Secondary outcomes are positive adjustment and QoL and health economics. Process measures are also included for the 


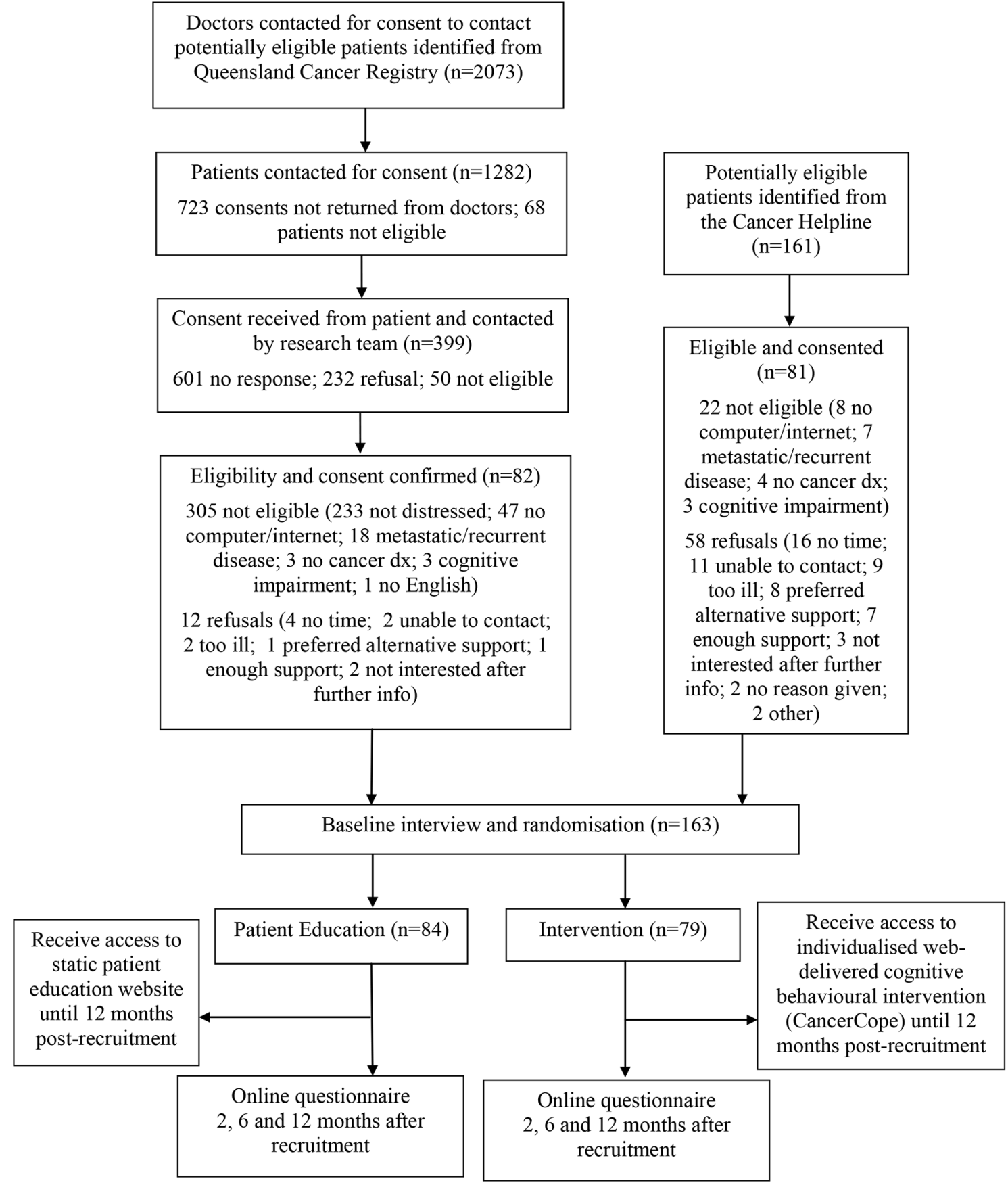

Figure 1 Flow chart of recruitment and intervention for phase II.

intervention arm. For participants recruited through the QCR, disease variables (eg, cancer grade, stage) are assessed through cancer registry records.

\section{Background variables}

Background variables include sociodemographic and disease variables (eg, cancer site and stage, medical treatments received, use of alternative therapies). We also assess previous (past month) and current use of support services and reassess use of such services at subsequent self-report assessments. This will allow us to control for any background effects in our analyses and contribute data to the economic analysis.

\section{Primary outcomes}

Psychological distress: The BSI $18^{27}$ assessed psychological distress through three subscales of depression, anxiety and somatisation, and also yields an overall distress score, the GSI. Higher scores indicate higher psychological distress.

Cancer-specific distress: The Impact of Event Scale $(\text { IES })^{28} 29$ assessed cancer-specific distress. The IES has two subscales that measure the extent to which participants are experiencing intrusive thoughts about cancer and avoiding thinking about cancer. Epping-Jordan $e t a \vec{l}^{30}$ suggest that intrusion and avoidance are more sensitive 
measures of psychological distress after a cancer diagnosis than generalised distress measures. Higher scores indicate higher cancer-specific distress.

Unmet supportive care needs: The Supportive Care Needs Survey Short Form 34 is a 34-item survey assessing the need for help for patients with cancer over the last month across five domains: psychological, health systems and information, patient care and support, physical and daily living and sexuality needs. Higher scores reflect higher support needs. It has well demonstrated reliability and validity in cancer populations. ${ }^{31}$

\section{Secondary outcomes}

Positive adjustment: Positive adjustment was measured with a 21-item Post-traumatic Growth Inventory assessing perceived positive life change occurring after a diagnosis of cancer. ${ }^{32}$ Domains assessed include strengthened relationships, appreciation of life, personal strength, new priorities and spiritual/religious growth. Higher scores indicate greater positive life changes. This scale is widely used in cancer populations. ${ }^{33}$

QoL and health economics: The Assessment of Quality of Life-8D (AQoL-8D) ${ }^{34}$ is a 35 -item scale assessing QoL on eight dimensions including: independent living, relationships, mental health, self-worth, happiness, coping, pain and sensory perception, and allows a simple global utility score to be calculated. Higher scores indicate better functioning and QoL. The AQoL-8D is the primary outcome instrument for the economic appraisal, has excellent psychometric properties and has been used in over 80 trials in Australia. We also obtain self-report data about visits to primary healthcare providers (general practitioners (GPs), psychologists) and use of prescription medications on the Pharmaceutical Benefits Scheme at baseline and at 12-month follow-up. These data will be used in an alongside trial cost-utility analysis of the trial.

\section{Process variables}

Participants in the CancerCope arm complete three process measures at the end of the intervention (2 months) and again at the end of follow-up (12 months).

Internet evaluation and utility questionnaire: assesses patients' experiences and perceptions of an internet intervention. $^{35}$ 36 This measure includes ease of use, convenience, engagement, enjoyment, layout, privacy, satisfaction and acceptability, and perceptions of the web programme material in terms of usefulness, comprehension, credibility, likelihood of returning, mode of delivery and helpfulness.

Internet intervention adherence questionnaire: identifies obstacles and barriers that interfere with using internet intervention programmes. ${ }^{35} 37$

Internet impact and effectiveness questionnaire: assesses patients' perceptions of the internet intervention in terms of the programme's effectiveness in resolving their targeted health condition. Perceived impact is measured in terms of helpfulness, knowledge gains, treatment effectiveness for self, treatment effectiveness for others, long-term effectiveness, QoL, mood, physical activity, family relationships, peer relationships, social activity, school/work attendance, school/work performance, treatment implementation, goal orientation, confidence in ability to manage the health condition, relapse prevention and service reduction. ${ }^{35} 36$

\section{Statistical analyses}

The study is a multivariate, two-condition RCT with repeated measures across time. We will examine this design using a multilevel model in which measurement occasions (level 1) are nested within persons (level 2) and in which programme differences are represented as a fixed effect at level 2 whose interaction with time represents differential adjustment and distress trajectories for the two groups. This approach to longitudinal designs is widely applied to contemporary intervention research. A key advantage of this approach is flexibility in dealing with missing data owing either to random or non-random attrition. Further, subgroups of patients who respond differentially to the programme will be identified using growth mixture modelling that is a related longitudinal procedure that identifies cases with similar distress and adjustment trajectories across time. ${ }^{38}$

A post hoc power calculation based on 79 people in the CancerCope Intervention and 84 in the Patient Education arm (163 in total) means that our study cohort will provide $89 \%$ power to detect a medium effect size $(0.5)$ with a significance level (alpha) of 0.05 using a two-sided two-sample t-test.

The cost-utility analysis will be undertaken from the perspective of costs to the health system for primary healthcare associated with psychological distress. Resource use, such as GP, specialist and psychology visits, will be multiplied by the unit costs for those services. The average cost per patient per month for each class of pharmaceutical will be included, along with the cost of the intervention. Costs will be summed for the intervention and the control groups. The AQoL-8D will be scored with the Australian value set and QALYs estimated as the area under the curve. The costs and QALYs in the intervention and control groups will be compared and the additional cost per QALY gained estimated. Sensitivity analysis, and where feasible subgroup analyses, will be undertaken to identify key parameters and populations with the greatest effect on results.

\section{ETHICS AND DISSEMINATION \\ Ethics}

Ethical approval was obtained from the Griffith University Human Research Ethics Committee (approval: PSY/70/13/HREC) and the Metro South Human Research Ethics Committee (HREC/13/QPAH/601). All participants provide informed consent prior to taking part in the study. 
Table 2 Sample sociodemographic characteristics and self-reported cancer history and support access for participants in phase II $(n=163)$

\begin{tabular}{ll}
\hline Variable & $\mathbf{n}(\%)$ \\
\hline Sex & \\
Male & $52(32)$ \\
Female & $111(68)$
\end{tabular}

Age group at diagnosis (years)

\begin{tabular}{|c|c|}
\hline $18-49$ & $43(26)$ \\
\hline $50-69$ & $91(56)$ \\
\hline $70+$ & $29(18)$ \\
\hline Mean age (years) & 57.3 \\
\hline \multicolumn{2}{|l|}{ Partner } \\
\hline Yes & $123(75)$ \\
\hline No & $40(25)$ \\
\hline
\end{tabular}

\section{Education}

\begin{tabular}{|c|c|}
\hline University & $44(27)$ \\
\hline Trade/certificate & $56(34)$ \\
\hline Senior high & $17(10)$ \\
\hline Less than senior & $46(28)$ \\
\hline \multicolumn{2}{|l|}{ Employment } \\
\hline Employed & $56(34)$ \\
\hline Retired & $46(28)$ \\
\hline Other & $61(38)$ \\
\hline \multicolumn{2}{|l|}{ Income } \\
\hline$<\$ 40000$ & $52(32)$ \\
\hline$\$ 40000-80000$ & $46(28)$ \\
\hline$\$ 80000-130000$ & $32(20)$ \\
\hline$\$ 130000+$ & $24(15)$ \\
\hline Not answered & $9(5)$ \\
\hline \multicolumn{2}{|l|}{ Cancer type } \\
\hline Colorectal & $60(37)$ \\
\hline Breast & $42(26)$ \\
\hline Melanoma & $29(18)$ \\
\hline Other & $32(19)$ \\
\hline \multicolumn{2}{|c|}{ Time to recruitment after diagnosis } \\
\hline 0-3 months & $48(29)$ \\
\hline 4-6 months & $58(36)$ \\
\hline $6-12$ months & $38(23)$ \\
\hline More than 12 months & $19(12)$ \\
\hline Median time (days) & 139 \\
\hline \multicolumn{2}{|l|}{ Treatment type } \\
\hline Had surgery & $139(85)$ \\
\hline Had chemotherapy & $68(42)$ \\
\hline Had radiation therapy & $20(12)$ \\
\hline Had hormone therapy & $1(1)$ \\
\hline Had other treatment & $17(10)$ \\
\hline
\end{tabular}

Continued

\begin{tabular}{ll} 
Table 2 Continued & \\
\hline Variable & $\mathbf{n}(\%)$ \\
\hline Access psychological care (yes) & $20(12)$ \\
Psychiatrist & $5(3)$ \\
Psychologist & $15(9)$ \\
Counsellor & $4(2)$ \\
Access support (yes) & \\
Doctor & $118(72)$ \\
Social worker & $20(12)$ \\
Nurse/other health professionals & $73(45)$ \\
Family/friends & $143(88)$ \\
Internet & $87(53)$ \\
Library & $4(2)$ \\
Books/brochures from doctor & $85(52)$ \\
Books/brochures from family & $18(11)$ \\
Cancer Helpline & $81(50)$ \\
Support group & $15(9)$ \\
CCQ counselling service & $17(10)$ \\
Other counselling service & $4(2)$ \\
Other support & $49(30)$ \\
\hline
\end{tabular}

*People can access more than one psychological care service. $\mathrm{CCQ}$, Cancer Council Queensland.

\section{Patients}

Recruitment for phase II commenced through the QCR in April 2015 and through the Cancer Helpline in November 2015, and was completed in May 2016 with follow-up ongoing. A total of 163 participants were recruited into the study. For QCR recruitment, once eligibility was confirmed, $87.2 \%$ agreed to participate. Of those eligible patients identified through the Cancer Helpline, $58.3 \%$ agreed to participate.

The main reasons for ineligibility were: participants not being distressed, no access to internet/computer and metastatic or recurrent disease. For example, over $58 \%$ of participants who provided consent through the QCR recorded a distress level of less than 4 on the DT. ${ }^{9}$ Main reasons for refusal included: no time to participate,

Table 3 Unmet supportive care needs for participants in phase II $(n=163)$

\begin{tabular}{|c|c|c|}
\hline Need domain & $\begin{array}{l}\% \text { reporting low, } \\
\text { moderate or high } \\
\text { need }\end{array}$ & $\begin{array}{l}\text { \% reporting } \\
\text { moderate or high } \\
\text { need }\end{array}$ \\
\hline $\begin{array}{l}\text { Physical and daily } \\
\text { living }\end{array}$ & 77.9 & 55.2 \\
\hline Psychological & 88.3 & 74.2 \\
\hline Patient care/support & 40.5 & 24.5 \\
\hline Sexuality & 36.2 & 23.3 \\
\hline $\begin{array}{l}\text { Health system/ } \\
\text { information }\end{array}$ & 54.0 & 41.7 \\
\hline
\end{tabular}


Table 4 Top 10 unmet supportive care needs by low, moderate or high need and moderate or high for participants in phase II $(n=163)$

\begin{tabular}{|c|c|c|}
\hline Need item & $\begin{array}{l}\% \text { reporting low, moderate } \\
\text { or high need }\end{array}$ & Need domain \\
\hline \multicolumn{3}{|l|}{ Low, moderate or high need } \\
\hline Uncertainty about the future & 71.2 & Psychological \\
\hline Concerns about the worries of those close to you & 67.5 & Psychological \\
\hline Fears about the cancer spreading & 66.3 & Psychological \\
\hline Worry that the results of treatment are beyond your control & 64.4 & Psychological \\
\hline Learning to feel in control of your situation & 59.5 & Psychological \\
\hline Lack of energy/tiredness & 57.7 & Physical and daily living \\
\hline Not being able to do the things you used to do & 56.4 & Physical and daily living \\
\hline Feeling down or depressed & 55.8 & Psychological \\
\hline Feelings of sadness & 54.0 & Psychological \\
\hline Anxiety & 53.4 & Psychological \\
\hline \multicolumn{3}{|l|}{ Moderate or high need } \\
\hline Uncertainty about the future & 49.7 & Psychological \\
\hline Fears about the cancer spreading & 44.8 & Psychological \\
\hline Concerns about the worries of those close to you & 44.2 & Psychological \\
\hline Worry that the results of treatment are beyond your control & 42.3 & Psychological \\
\hline Lack of energy/tiredness & 37.4 & Physical and daily living \\
\hline Not being able to do the things you used to do & 35.0 & Physical and daily living \\
\hline Work around the home & 33.7 & Physical and daily living \\
\hline Learning to feel in control of your situation & 31.9 & Psychological \\
\hline Anxiety & 29.5 & Psychological \\
\hline Feelings of sadness & 28.8 & Psychological \\
\hline
\end{tabular}

participation impaired by illness or treatment, preferred alternative support (eg, face-to-face counselling rather than computer-based support), already had enough support from health professionals and/or friends and family, did not need support and not interested after receiving further information.

\section{Background and support use}

The sociodemographic characteristics, cancer history and support use of participants at baseline are reported in table 2. The median time since diagnosis at baseline was 139 days. Twelve per cent were currently receiving specialised psychological care. Since their cancer diagnosis, $88 \%$ of participants reported receiving support from family and friends, $72 \%$ from a doctor, $53 \%$ from the internet, $52 \%$ from books or brochures from a doctor, $50 \%$ from the Cancer Helpline and $45 \%$ from a nurse or other health professionals.

\section{Psychological distress}

A total of $50.3 \%$ participants met caseness on the BSI 18 for clinically significant psychological distress (as indicated by a score of $\geq 57$ for either total GSI t-score or two subscale t-scores) ${ }^{25}$ The mean scores for each dimension in the BSI 18 were $4.7(\mathrm{SD}=4.0)$ for somatisation, 4.4
$(\mathrm{SD}=4.2)$ for anxiety and $4.0(\mathrm{SD}=4.5)$ for depression, with a mean GSI score of $13.1(\mathrm{SD}=10.8)$.

\section{Supportive care needs}

Table 3 provides descriptive statistics for the supportive care needs domains. A high proportion $(88.3 \%)$ of participants reported some unmet need (low, moderate or high) in the psychological domain, with $74.2 \%$ reporting moderate or high need in this domain. This was followed by physical and daily living ( $77.9 \%$ and $55.2 \%)$, health system/information $(54.0 \%$ and $41.7 \%)$, patient care/ support $(40.5 \%$ and $24.5 \%)$ and sexuality $(36.2 \%$ and 23.3\%) domains. The top 10 unmet needs are listed in table 4 for low, moderate or high need and moderate or high need, with the majority of these in the psychological domain.

\section{DISCUSSION}

As in previous studies, ${ }^{14} 39$ the area of greatest unmet need in this cohort of patients with cancer was in the psychological domain and, in particular, fears about cancer recurrence. Needs around feeling depressed or anxious, tiredness, loss of control and concerns about family were also highly prevalent. This may be expected in a patient 
population that have been screen detected as distressed (although only $50 \%$ reached caseness on retesting). However, we note that of these patients, only $12 \%$ were receiving specialised psychological care on study entry. We propose that this emphasises the point that many distressed cancer patient do not seek or receive professional psychological care within the current cancer care system, ${ }^{40}$ and that accessible care models are urgently needed. ${ }^{41}{ }^{42}$ Moreover, this need will only increase as the cancer burden escalates in the future. ${ }^{434}$

Once completed, this trial will provide recommendations about the efficacy of web-based cognitive behavioural interventions to facilitate better psychosocial adjustment and mental health for people with cancer. We note that recruitment rates were much lower for Cancer Helpline callers compared with those approached from the cancer registry. This suggests that Helpline callers who have already accessed telephone-based support may feel their support needs to be met or may be disinclined to use an alternative remote access service. However, the high recruitment rate for patients from the QCR suggests the web-based approach is acceptable for patients who are not already linked in to community-based support services. To our knowledge, there is no readily available online psychological intervention that directly targets the needs of distressed patients with cancer in the Australian setting. The project will provide an evidence-based, practical and applied approach to psychological intervention for people with cancer that can be rapidly translated into the population, and improve health outcomes by delivering an 'on demand' web-based psychological therapy service targeted to the needs of this group.

\section{Author affiliations \\ ${ }^{1}$ Menzies Health Institute Queensland, Griffith University, Gold Coast, Australia \\ ${ }^{2}$ Cancer Council Queensland, Brisbane, Australia \\ ${ }^{3}$ Prostate Cancer Foundation of Australia, Sydney, Australia \\ ${ }^{4}$ Health and Wellness Institute, Edith Cowan University, Perth, Australia \\ ${ }^{5}$ Institute for Resilient Regions, University of Southern Queensland, Springfield, Australia \\ ${ }^{6}$ University of Virginia, Charlottesville, Virginia, USA \\ ${ }^{7}$ School of Medicine, Griffith University, Australia \\ ${ }^{8}$ University of the Sunshine Coast, Sippy Downs, Australia \\ ${ }^{9}$ School of Public Health and Social Work, University of Technology, Kelvin Grove, Australia \\ ${ }^{10}$ Menzies School of Health Research, Brisbane, Australia \\ ${ }^{11}$ School of Social Science, The University of Queensland, St Lucia, Australia}

Correction notice This paper has been amended since it was published Online First. Owing to a scripting error, some of the publisher names in the references were replaced with 'BMJ Publishing Group'. This only affected the full text version, not the PDF. We have since corrected these errors and the correct publishers have been inserted into the references.

Acknowledgements We gratefully acknowledge the support of Dr Cindy Mervin, Dr Lizbeth Kenny, Dr lan Irving and Dr Euan Walpole as associate investigators, and of Ms Leonie Young as a consumer advisor.

Contributors SKC, JD, LR and FT developed the study concept and aims and initiated the project. All other authors assisted in further development of the protocol. SKC was responsible for drafting the manuscript. SKC, SC, LN and FT implemented the protocol and oversaw collection of the data. All authors contributed to the final manuscript.
Funding This project was funded by the Australian National Health and Medical Research Council and Cancer Council Queensland (ID APP1056756). PY is funded by a National Health and Medical Research Council Early Career Fellowship (ID APP1054038).

\section{Patient consent None.}

Ethics approval Griffith University Human Research Ethics Committee (Approval: $\mathrm{PSY} / 70 / 13 / \mathrm{HREC}$ ) and the Metro South Human Research Ethics Committee (HREC/13/ QPAH/601).

Provenance and peer review Not commissioned; peer reviewed.

Data sharing statement The datasets generated during and/or analysed during the current study are not publicly available due to ethical requirements but are available from the corresponding author on reasonable request.

Open Access This is an Open Access article distributed in accordance with the Creative Commons Attribution Non Commercial (CC BY-NC 4.0) license, which permits others to distribute, remix, adapt, build upon this work non-commercially, and license their derivative works on different terms, provided the original work is properly cited and the use is non-commercial. See: http://creativecommons.org/ licenses/by-nc/4.0/

(C) Article author(s) (or their employer(s) unless otherwise stated in the text of the article) 2017. All rights reserved. No commercial use is permitted unless otherwise expressly granted.

\section{REFERENCES}

1. Australian Institute of Health and Welfare. Cancer in Australia: an overview 2014. Cancer series No 90 Cat no CAN 88. Canberra: AlHW, 2014.

2. Zabora J, BrintzenhofeSzoc K, Curbow B, et al. The prevalence of psychological distress by cancer site. Psychooncology 2001;10:19-28.

3. Helgeson VS, Snyder P, Seltman H. Psychological and physical adjustment to breast cancer over 4 years: identifying distinct trajectories of change. Health Psychol 2004;23:3-15.

4. Fall K, Fang F, Mucci LA, et al. Immediate risk for cardiovascular events and suicide following a prostate cancer diagnosis: prospective cohort study. PLoS Med 2009;6:e1000197.

5. Bill-Axelson A, Garmo H, Lambe M, et al. Suicide risk in men with prostate-specific antigen-detected early prostate cancer: a nationwide population-based cohort study from PCBaSe Sweden. Eur Urol 2010;57:390-5.

6. Fang F, Keating NL, Mucci LA, et al. Immediate risk of suicide and cardiovascular death after a prostate cancer diagnosis: cohort study in the United States. J Natl Cancer Inst 2010;102:307-14.

7. Canadian Association of Psychosocial Oncology. Standards of Psychosocial Health Services for persons with cancer and their families. Canada: CAPO, 2010.

8. National Breast Cancer Centre and National Cancer Control Initiative. Clinical practice guidelines for the psychosocial care of adults with Cancer. Camperdown, NSW: National Breast Cancer Centre, 2003

9. National Comprehensive Cancer Network. Clinical Practice guidelines in Oncology (NCCN guidelines). Distress Management Version 2.2013. , 2013.

10. Rankin NM, Barron JA, Lane LG, et al. Psychosocial oncology services in New South Wales. Aust Health Rev 2011;35:156-63.

11. Turner J, Kelly B, Clarke D, et al. A tiered multidisciplinary approach to the psychosocial care of adult cancer patients integrated into routine care: the PROMPT study (a cluster-randomised controlled trial). Support Care Cancer 2017;25:17-26.

12. Harrison JD, Young JM, Price MA, et al. What are the unmet supportive care needs of people with cancer? A systematic review. Support Care Cancer 2009;17:1117-28.

13. Hodgkinson $\mathrm{K}$, Butow $\mathrm{P}$, Hobbs KM, et al. Assessing unmet supportive care needs in partners of cancer survivors: the development and evaluation of the Cancer Survivors' Partners Unmet Needs measure (CaSPUN). Psychooncology 2007;16:805-13.

14. Sanson-Fisher R, Girgis A, Boyes A, et al. The unmet supportive care needs of patients with cancer. Cancer 2000;88:226-37.

15. Hardyman R, Hardy P, Brodie J, et al. It's good to talk: comparison of a telephone helpline and website for cancer information. Patient Educ Couns 2005;57:315-20.

16. Twomey C. Telephone contacts with a cancer nurse specialist. Nurs Stand 2000;15:35-8.

17. Steginga SK, Hutchison S, Turner J, et al. Translating psychosocial care guidelines Into action. Cancer Forum 2006;30:28-31. 
18. Ritterband LM, Gonder-Frederick LA, Cox DJ, et al. Internet interventions: in review, in use, and into the future. Prof Psychol 2003;34:527-34.

19. Ritterband LM, Thorndike FP. The further rise of internet interventions. Sleep 2012;35:737-8.

20. Barlow J, Wright C, Sheasby J, et al. Self-management approaches for people with chronic conditions: a review. Patient Educ Couns 2002;48:177-87

21. Willemse GR, Smit F, Cuijpers P, et al. Minimal-contact psychotherapy for sub-threshold depression in primary care. Randomised trial. Br J Psychiatry 2004;185:416-21.

22. Lovell K, Richards D. Multiple access points and levels of entry (MAPLE): Ensuring choice, accessibility and equity for CBT services. Behavioural and Cognitive Psychotherapy 2000;2000:379-91.

23. Hutchison SD, Sargeant $H$, Morris BA, et al. A community-based approach to cancer counselling for patients and carers: a preliminary study. Psychooncology 2011;20:897-901.

24. Chambers SK, Girgis A, Occhipinti S, et al. A randomized trial comparing two low-intensity psychological interventions for distressed patients with cancer and their caregivers. Oncol Nurs Forum 2014;41:E256-E266.

25. Zabora J, BrintzenhofeSzoc K, Jacobsen P, et al. A new psychosocial screening instrument for use with cancer patients. Psychosomatics 2001;42:241-6.

26. Altman DG, Schulz KF, Moher D, et al. The revised CONSORT statement for reporting randomized trials: explanation and elaboration. Ann Intern Med 2001;134:663-94.

27. Derogatis L, Lopez M. Brief Symptom Inventory 18: Administration, Scoring and Procedures Manual. Minneapolis: National Computer Systems Inc, 2000.

28. Horowitz M, Wilner N, Alvarez W. Impact of Event Scale: a measure of subjective stress. Psychosom Med 1979;41:209-18.

29. Weiss DS, Marmar CR. The Impact of Event Scale - Revised. In: Wilson JP, Keane TM, eds. Assessing Psychological Trauma and PTSD. New York: Guilford Press, 1997:399-411.

30. Epping-Jordan JE, Compas BE, Howell DC. Predictors of cancer progression in young adult men and women: avoidance, intrusive thoughts, and psychological symptoms. Health Psychol 1994;13:539-47.

31. Boyes A, Girgis A, Lecathelinais C. Brief assessment of adult cancer patients' perceived needs: development and validation of the 34- item Supportive Care Needs Survey (SCNS-SF34). J Eval Clin Pract 2009;15:602-6.

32. Tedeschi RG, Calhoun LG. The Posttraumatic Growth Inventory: measuring the positive legacy of trauma. J Trauma Stress 1996;9:455-71.

33. Stanton AL, Bower JE, Low CA. Posttraumatic growth after cancer In: Calhoun LH, Tedeschi RG, eds. Handbook of Posttraumatic Growth: Research and Practice. Mahwah, NJ, USA: Erlbaum, 2006:138-75.

34. Richardson J, lezzi A, Khan MA, et al. Validity and reliability of the Assessment of Quality of Life (AQoL)-8D multi-attribute utility instrument. Patient 2014;7:85-96.

35. Ritterband LM, Ardalan K, Thorndike FP, et al. Real world use of an internet intervention for pediatric encopresis. J Med Internet Res 2008;10:e16.

36. Thorndike FP, Saylor DK, Bailey ET, et al. Development and perceived utility and impact of an internet intervention for insomnia. $E \mathrm{~J}$ Appl Psychol 2008;4:32-42.

37. Ritterband LM, Borowitz S, Cox DJ, et al. Using the internet to provide information prescriptions. Pediatrics 2005;116:e643-e647.

38. Muthén $\mathrm{B}$, Brown $\mathrm{CH}$, Masyn $\mathrm{K}$, et al. General growth mixture modeling for randomized preventive interventions. Biostatistics 2002;3:459-75

39. Hall A, Lynagh M, Bryant J, et al. Supportive care needs of hematological cancer survivors: a critical review of the literature. Crit Rev Oncol Hematol 2013;88:102-16.

40. Holland J, Watson M, Dunn J. The IPOS New International Standard of Quality Cancer Care: integrating the psychosocial domain into routine care. Psychooncology 2011;20:677-80.

41. Chambers SK, Hutchison S, Clutton S, et al. Intervening to improve psychological outcomes after cancer: what is known and where next? Aust Psychol 2014;49:96-103.

42. Hutchison SD, Steginga SK, Dunn J. The tiered model of psychosocial intervention in cancer: a community based approach Psychooncology 2006;15:541-6.

43. Schofield P, Chambers S. Effective, clinically feasible and sustainable: Key design features of psycho-educational and supportive care interventions to promote individualised selfmanagement in cancer care. Acta Oncol 2015;54:805-12.

44. Watson M, Dunn J. The multidisciplinary art and science of cancer care: integrating psycho-oncology. Future Oncol 2016;12:2775-8. 